\title{
Android Printing: Towards On-Demand Android Development Employing Multi-Material 3-D Printer
}

This paper was downloaded from TechRxiv (https://www.techrxiv.org).

LICENSE

CC BY 4.0

SUBMISSION DATE / POSTED DATE

22-07-2021 / 26-07-2021

CITATION

Yagi, Satoshi (2021): Android Printing: Towards On-Demand Android Development Employing Multi-Material 3-D Printer. TechRxiv. Preprint. https://doi.org/10.36227/techrxiv.15034623.v1

$\mathrm{DOI}$

10.36227/techrxiv.15034623.v1 


\title{
Android Printing: Towards On-Demand Android Development Employing Multi-Material 3-D Printer
}

\author{
Satoshi Yagi, Yoshihiro Nakata, and Hiroshi Ishiguro
}

\begin{abstract}
In this paper, we propose the concept of Android Printing, which is printing a full android, including skin and mechanical components in a single run using a multi-material 3$D$ printer. Printing an android all at once both reduces assembly time and enables intricate designs with a high degrees of freedom. To prove this concept, we tested by actual printing an android. First, we printed the skin with multiple annular ridges to test skin deformation. By pulling the skin, we show that the state of deformation of the skin can be adjusted depending on the ridge structure. This result is essential in designing humanlike skin deformations. After that, we designed and fabricated a 3-D printed android head with 31 degrees of freedom. The skin and linkage mechanism were printed together before connecting them to a unit combining several electric motors. To confirm our concept's feasibility, we created several motions with the android based on human facial movement data. In the future, android printing might enable people to use an android as their own avatar.

Index Terms-Humanoid Robot Systems, Gesture, Posture and Facial Expressions, Soft Robot Materials and Design, Telerobotics and Teleoperation
\end{abstract}

\section{INTRODUCTION}

Recently, it has become possible for people to represent themselves remotely using teleoperated robots - whether it be for work presentations or experiencing faraway destinations through the eyes of a robot [1], [2]. Several teleoperated robots have already been developed, and these teleoperated robots can be used as an avatar to enable a variety of social participation. One crucial function of such robots is to convey the operator's presence to people interacting with the robot. Therefore, androids with very human-like appearances have been created to convey a clearer sense of presence. Alongside this, several fields of research have developed to realize human-like behavior within these androids.

However, there have been reports on the challenges robots face in conveying the remote operator's presence. For example, robots can perform or display only limited facial movements in actual conversation situations. Moreover, these robots often have a robot-specific appearance that causes decreased task efficiency and lower conversation frequency [3], [4]. While human-like robots are preferred for social roles due to their anthropomorphic physical appearance, the same appearance can lead to complex social expectations -

This research was partially supported by JST ERATO Grant Number JPMJER1401 and Grant-in-Aid for JSPS Fellows Grant Number 19J20127. Satoshi Yagi and Hiroshi Ishiguro are with Department of Systems Innovation, Graduate School of Engineering Science, Osaka University, Osaka 560-0043, Japan. Yoshihiro Nakata is with Department of Mechanical and Intelligent Systems Engineering, Graduate School of Informatics and Engineering, The University of Electro-Communications, Tokyo 182-8585, Japan (email:ynakata@uec.ac.jp)

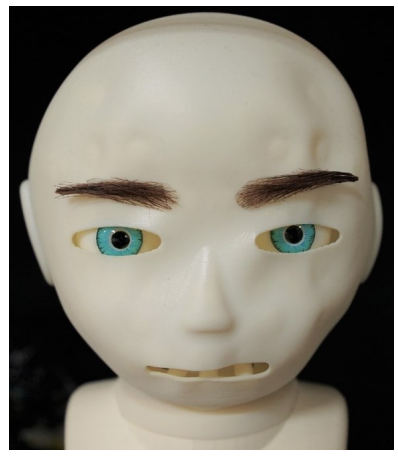

(a)

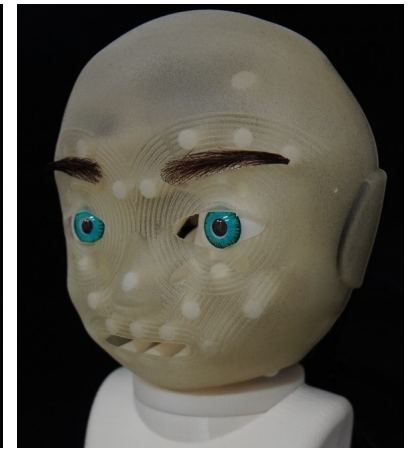

(b)
Fig. 1. Androids printed all at once using a multi-material 3-D printer except for the actuators. Note that we added eyebrows and irises after printing; (a) Android with soft white skin; (b) Android with transparent soft skin. The rigid links behind the skin are showing through it.

if it looks like a human, it should behave like one [5]. Thus, there is a need for improved methods in teleoperated robots to convey better human-like presence.

In light of these insights, we have been working on the development of an android that has a human-like appearance, as well as researching the presence of these robots in society [6]. Our goal is to create an android that can be used as an avatar of the operator, capable of conveying the operator's presence to a remote location by precisely reproducing the operator's appearance and behavior. If such androids can be realized, they can be used to facilitate more natural interaction with people in remote places. However, it has not been easy to create a personalized android. A significant reason for the difficulty in creating androids lies in the manufacturing process. Most of the androids developed so far have a silicone skin driven by actuators (pneumatic cylinders or electric motors) via cables and links. There is a shell structure made of plastic or other material behind the skin that allows the skin to retain its human shape, with mechanisms built inside the shell. The skin is made by silicone through a molding process using a mold taken from a person. The shell and mechanical parts are manufactured separately and are then assembled to make an android. As this traditional manufacturing process is timely and costly, it has not been easy to develop customized androids for common use.

In recent years, remarkable advances in 3-D printing technology and materials have allowed new possibilities for robot development. Since 3-D printers do not require molds that are usually used in conventional manufacturing, modification of product designs for 3-D printing is easy. 
Application of 3-D printing is also not only limited to the manufacture of mechanical parts - it can also be used to print human organs. Integrated manufacturing by 3-D printing is effective in reducing the number of parts. Thus, using a 3-D printer to create the face and body parts of humanoid robots can reduce the number of mechanical components [7], [8]. Some 3-D printers have multi-material printing functions that can print both rigid and flexible materials simultaneously, and have been used to develop robots that interact with humans - such as creating soft skin for an arm robot [9] or a soft actuator for a legged robot [10].

However, this paper is the first to 3-D print an android's facial skin and internal mechanisms simultaneously. Even recent android heads have to be assembled with partially printed mechanical parts with a separate applied silicone skin created using a facial mold [11]. Thus, this research aims to develop a new method of android printing technology using multi-material 3-D printing to print an android simultaneously - including the skin and its whole mechanical components. The advantages of using 3-D printing for the fabrication of androids are: (1) it reduces assembly time and cost by simultaneously molding both flexible skinlike material and rigid material into skeleton; and (2) the design of the individual faces can be easily modified without creating molds for making skin.

In this paper, we present a printed android head that we developed (see Fig. 1) to verify the feasibility of android printing. To achieve the android's human-like appearance, we proposed a specific skin structure design to achieve the desired skin deformation. To confirm its effectiveness through experiments, we connected 31 electric motors to the rigid links in the printed android head and operated them. To test the android's use as an avatar, we sent commands to the motors moving the android based on deformation data of a human face captured using motion capture. Lastly, we discuss the experiment results, including discussion on what is necessary to realize printing a complete android - including all elements and actuators - all at once.

\section{CONCEPT OVERVIEW}

The face is one of the most important parts of an android. Our design blueprint involves printing both soft face skin and rigid link mechanisms simultaneously using a multi-material 3-D printer. We then later attached an actuator unit and rotate rigid links to deform the skin. However, a problem of our design is the following - the orbicularis oris muscle around the human mouth is a ring-shaped structure which pulls the skin surface. This deformation mechanism is different to our designed link mechanism that pulls a specific point on the skin. To alleviate this, we introduce the ridged skin design for a natural facial expression.

The concept of our android head is to build the facial structure by manufacturing both the soft skin and its actuating mechanisms at once through the use of multi-material 3-D printing. The face's skin surface is designed based on a 3-D CG model of a human face and is printed with a flexible material. For example, 3-D printing materials such

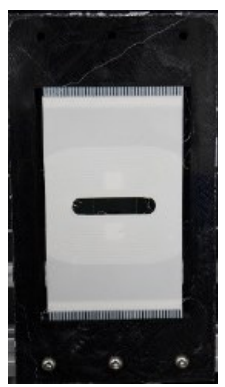

(a)

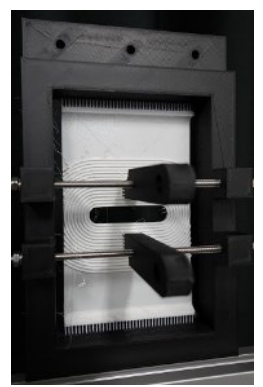

(b)

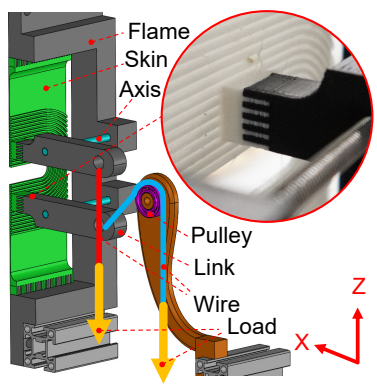

(c)
Fig. 2. The figures show experimental setup for the printed skin deformation test; (a) Front side photograph, the white part is the printed skin, and both the top and bottom end are connected to the fixed frame (the black part); (b) back side photograph, two rigid links are connected to the skin for deformation; (c) illustration of the experimental setup. The enlarged photograph shows the connection surface between the rigid link (the black part) and the soft skin (the white part) printed in a layered structure to strengthen the connection.

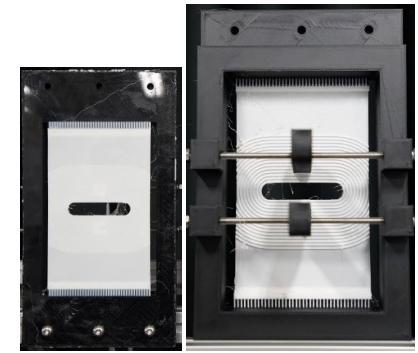

(a)

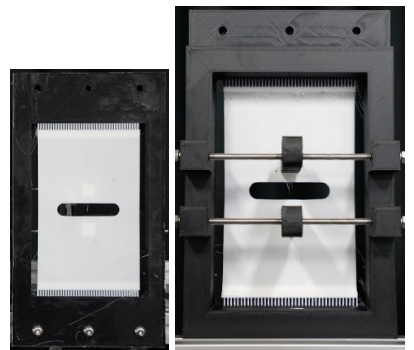

(b)
Fig. 3. The photographs of the pilot test models; (a) the skin with ridges on the surface around the hole; (b) the skin without ridges on the surface.

as TPU150 and Agilus30 were used as they are rubber-like materials that can be greatly deformed with force, but still return to its original shape when the force is released. The skeleton and links were printed with rigid materials like ABS and Acrylic, which have a relatively higher rigidity than flexible materials. Fig. 2 shows the pilot test model. This model was created by imitating a human-like mouth and eyes (Fig. 2 (a)). TPU150 was used as the flexible material (white), and ABS was used as the rigid material (black) on an Ultimaker S5 3-D printer (Ultimaker). The size of the white skin is $126 \mathrm{~mm}$ (height) by $80 \mathrm{~mm}$ (width) with a hole of $40 \mathrm{~mm}$ by $5 \mathrm{~mm}$ radius in the center. The soft skin part was printed with a main thickness of $0.5 \mathrm{~mm}$ to make it stretch well. Rigid links were attached on both the upper and lower sides of the hole. Torques were applied to each rigid link to deform the surface enough to form the open-mouth shape (Fig. 2 (b)). Fig. 2 (c) shows an overview of the experimental setup for applying torque to the model. To apply torque, we tied a wire to the end of each link before hanging a weight on the wire. The $\mathrm{x}$-axis and z-axis were set as shown in Fig. 2 (c). Both rigid and flexible materials can be printed with a single printer at the same time and the connection surface between those two materials do not require gluing. In order to strengthen the connection between those two materials, we have adopted a two-materials layered structure printing 


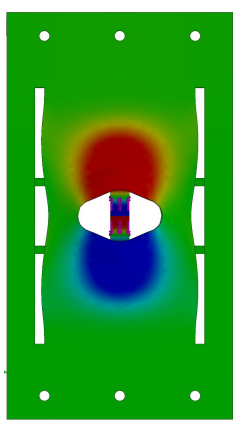

With ridges

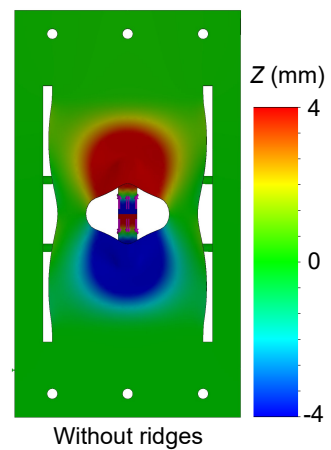

(a)

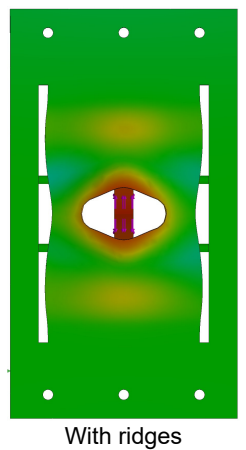

With ridges

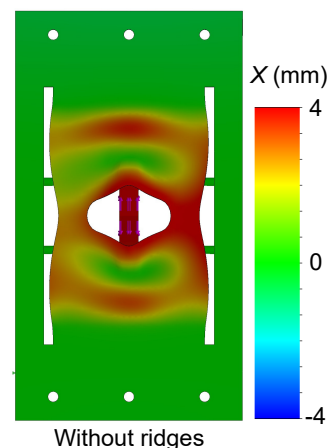

(b)
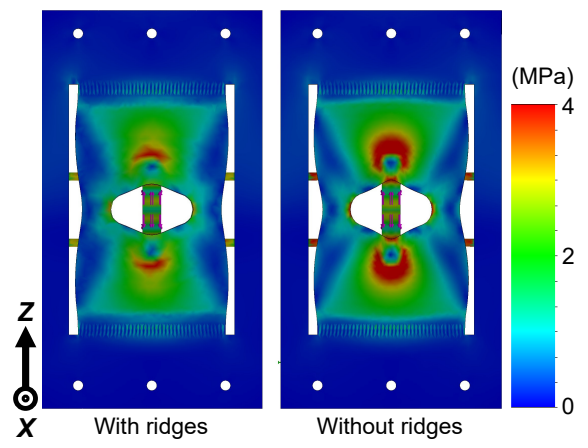

(c)

Fig. 4. Simulation results; (a) the deformation in z-axis direction; (left) the skin with ridges; (right) the skin without ridges. (b) the deformation in x-axis direction; (left) the skin with ridges; (right) the skin without ridges. (c) the distribution of von mises stress; (left) the skin with ridges; (right) the skin without ridges. Note that what we see in the hole is the back side links.

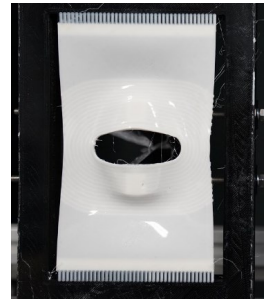

(a)

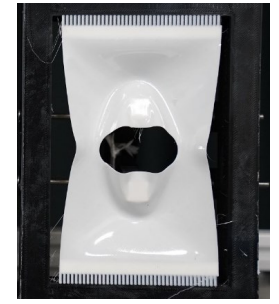

(b)

Fig. 5. Experimental results; (a) the skin with ridges on the surface around the hole; (b) the skin without ridges on the surface.

as shown in Fig. 2 (c).

For a comparison of the skin designs, we prepared two types of skins. For the first type, we designed additional 1 $\mathrm{mm}$ ring-shaped thickness ridges around the hole (as shown in Fig. 3 (a)), to make the shape more human-like when pulled by the rigid link. And the second type was designed without any ridges is shown in Fig. 3 (b).

Before printing the models, we ran a simulation of the skin deformation analysis. As simulation parameters, we set the Young's modulus at $1.18 \mathrm{MPa}$ and the Poisson's ratio at 0.45 for the skin. Additionally, the Young's modulus was set at 25.0 MPa and the Poisson's ratio at 0.36 for the rigid material of black body. Every link was applied a $0.8 \mathrm{Nm}$ torque in the direction that opens the hole.

The simulation results when stretching the mouth hole are shown in Fig. 4. Each result shows deformations of the skin with ridges around the hole (left) v.s. the flat skin without ridges (right). The result of the deformation in z-axis in Fig. 4 (a) shows that both the ridged and flat skin deformed in vertical direction to open the hole the same way. On the other hand, the result of the deformation in $\mathrm{x}$-axis in Fig. 4 (b) shows a problem that is caused by using the flat skin. As evident in the flat skin in Fig. 4 (b) right, the parts next to the mouth hole was also deformed when rigid links were applied to torques to open the hole. Also, due to the stiffness at connection surfaces between the links and the skin, unnatural swellings were caused in the parts further away from the

mouth hole. The result of the distribution of von mises stress are shown in Fig. 4 (c). It also shows the larger areas of highstressed skin parts above and below the hole at the flat skin.

After we confirmed the validity of our concept through simulation analysis, we printed the test models. Fig. 5 shows a comparison of the actual model's deformations with the ridged versus flat skin. When we applied a $0.5 \mathrm{Nm}$ torque to each rigid link, the flat skin could open the hole bigger than that of the ridged skin, but unnatural swellings were formed above and below the mouth hole. In contrast, we can see that the ridged skin was deformed naturally. In addition, noncontinuous stress around the hole edge caused the hole edge to bend out of shape as shown in Fig. 4 (c).

Through the simulation and the actual pilot test models, we confirmed that the multi-material 3-D printing design with a soft skin and rigid link mechanisms can be applied for android head printing. We also discovered that we can fix the skin deformation more naturally by adjusting the backside skin thickness with ridges.

\section{3-D PRINTED ANDROID HEAD}

In order to confirm the feasibility of android printing, we printed the android head, which has a complicated structure and requires a large number of actuators. During the development, the skin and links were printed simultaneously. After that, electric motors for driving were connected to the end of the links manually.

Fig. 6 (a) shows the CAD diagram of the developed android head. The actual machines of Fig. 1 (a) and (b) were created based on this CAD data. In order to more easily see the position of the eyes and eyebrows, we attached eyebrows and irises to the face. The main parameters are shown in Table I.

The hardware of the android head is mainly composed of three elements: a soft skin with grooves, an actuator unit, and a rigid link mechanism. The actuating mechanism is explained using the orange area of Fig. 6 (b). When an electric motor at an actuator unit rotates in some direction, the rigid link that connects the skin with the motor axis also inclines to pull the skin. Fig. 6 (c) shows three representative 
sectional views of moving points where red arrows point, which we call connection points in this paper, between rigid and flexible materials. In addition, the back of the head was created separately so that it could be removed when the actuator unit was attached into the head. The control board and power supply were installed externally. All components were designed in SolidWorks and printed using VeroPureWhite (rigid material) and Agilus30Clear/White (flexible material) on a Stratasys J750 3-D printer (Stratasys).

Fig. 7 (a) shows a view of the facial skin from the inside. We created $1.8 \mathrm{~mm}$ deep grooves (shown in yellow areas and red edges) in the face skin with a thickness of $3 \mathrm{~mm}$. Throughout, we referred to the shape of the human facial muscles. The thickness of the facial skin and the groove depth were determined on the condition that the 3-D printer could print them and that the skin would not break during the experiment.

An important function for android robots is to have real human-like appearance and movements. Agilus30 was used as the skin material for the android head. This material stretches up to $200 \%$ when pulled and quickly returns to the original shape when the force is released. Due to the transparent or white color, it is possible to paint it to recreate real human skin color and texture like in Fig. 7 (b) (left: before painting, right: after painting).

As each person has a different facial shape, facial movements also differ from person to person. In the field of character animation, a rigging process is required to perform natural facial expressions. Thus, for the fabrication of an android face, it is also required that the connection points on the skin can be changed easily. There is almost no need to assemble components except for the actuator unit in the android printing design process, which allows for a high density of connection points to be placed. Thus, firstly we designed the android with as many connection points as possible on the entire face as shown in Fig. 7 (c). We also referred to the Action Unit of the Facial Action Coding System by Ekman et al. [12]. We implemented 31 connection points in the android head. And then we created a smooth rigid link body from the connection point to each motor's axis of the actuator unit.

In order to easily and quickly build an android, it is necessary to reduce the number of parts. In our proposed method, we assembled the actuator unit in which multiple motors are lined up and are attached to the multi-material printed part. With this design method, we can use the same actuator unit design regardless of a person's face shape. With reference to the torque of the motor that drives the skin of our past developed android [13], we selected small high-power carbon brushes motors (Pololu, stall torque $0.15 \mathrm{Nm}$ ) for electric motors, DRV8801 (Pololu) for a motor driver, and LPC1768 (Mbed) for a micro-controller. PWM signals are sent to the electric motors in $120 \mathrm{~Hz}$ by using feed forward control.

\section{TABLE I}

MAIN PARAMETERS OF THE ANDROID HEAD.

\begin{tabular}{ccccc} 
Height & Width & Depth & Mass & DoF \\
\hline $127 \mathrm{~mm}$ & $113 \mathrm{~mm}$ & $112 \mathrm{~mm}$ & $558 \mathrm{~g}$ & 31
\end{tabular}

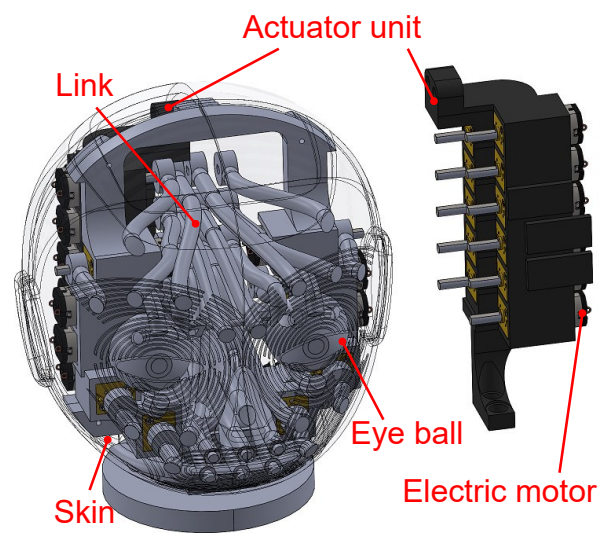

(a)

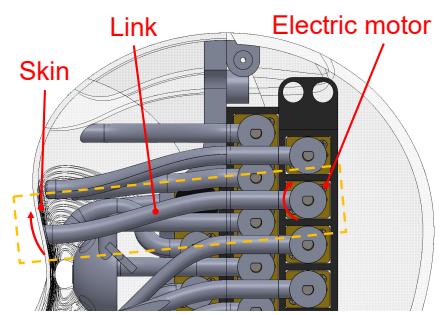

(b)

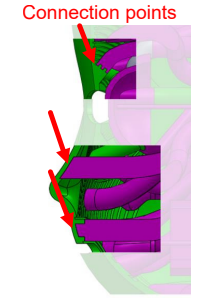

(c)
Fig. 6. Three-dimensional computer aided design image of the android head; (a) the whole structure; (b) the details of the link mechanism for actuating skin; (c) sectional views of connection points between flexible (green) and rigid (purple) materials.

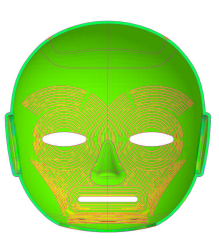

(a)

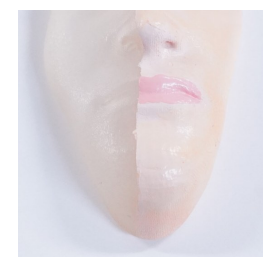

(b)

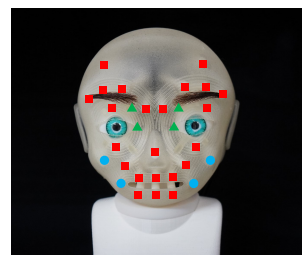

(c)
Fig. 7. (a) The grooved facial skin. The green part is $3.0 \mathrm{~mm}$ thickness and the yellow areas surrounded by red edges are $1.8 \mathrm{~mm}$ deep grooves. (b) The painted skin. (c) 31 connection points of the android head. Red markers (square) pull the skin vertically, blue markers (circle) rotate, and green markers (triangle) incline the eye balls.

\section{RESULT}

In this section, we show the result of the android head's facial expression. We prepared human facial expression data for facial expression generation by the android head. Except for eyeball movements, we recorded a time series of facial movements performed by the author using motion capture. We put motion capture markers at the same positions as the android head's connection points on the author's face, and then obtained the time series vertical displacement $y(t)$ from the recorded data.

The input value to each connection point $u(t)$ was calculated proportional to the displacement $y(t)$ as following Eq. 1. Here $K$ is the motor power limitation. $y_{t h r}$ was determined as $5 \mathrm{~mm}$. Furthermore, inputs to the eyeballs were set to 0 . Due to the limitation of motor speeds, we reduce the signal rate from 120 to $40 \mathrm{~Hz}$. Thus, the robot expressed 3 times 
slower movements than the actual movement speed of the human.

$$
u(t)= \begin{cases}K & \left(y(t)>y_{t h r}\right) \\ K \frac{y(t)}{y_{t h r}} & \left(y_{t h r} \geq y(t) \geq-y_{t h r}\right) \\ -K & \left(y(t)<-y_{t h r}\right)\end{cases}
$$

Fig. 8 shows three examples of the facial expression. The original author's facial expressions are indicated in the left. The android head's expressions are indicated in the right. To make it easier to see the differences between the facial expressions, we printed the android head with white color (Fig. 1 (a)). The facial expression (a) represents smiling. The feature is that the corner of the mouth is raised. Even in the android head, we can see the raised corner of the mouth. The facial expression (b) looks disgusted. The feature is that the mouth is open and the chin is down. Also in the android head, we can see the open mouth shape and the lowered jaw. The facial expression (c) looks neutral. In the android head, we can see that the mouth is slightly open as well.

\section{DISCUSSION}

The basic idea of our concept of android printing is to print the skin, skeleton, mechanical parts, and even the actuators of an android simultaneously using a multi-material 3-D printer. As the first validation of the feasibility of android printing, we created an android head by printing the soft skin and rigid mechanical parts, which is particularly complicated and important in the human body, before confirming facial movements by actuating electric motors. In the traditional development process for androids, the skin is molded by pouring silicone into a face mold, and the internal skeleton and mechanical parts are separately designed and assembled. The advantage of building an android head with a 3-D printer is that people can easily change the design according to human characteristics by modifying the $\mathrm{CG}$ data. This will reduce production time and costs, and will make it easier for people to use an android as their avatar. The design process using CG data also enabled the android head to be mounted to 31 actuators, even though the size was significantly smaller than the traditional android head.

For the head, especially the face, it is important that there is natural deformation when the actuator is driven in order to realize a human-like facial expression. Previous research has mainly focused on the investigation of the motion patterns and the internal mechanisms [14], [15], [16]. However, human facial skin has different thickness in each part, and complex deformation occur due to the coordinated movement of multiple facial muscles under the skin. Therefore, in this paper, a natural facial deformation can be created by designing a structure that has ridges or grooves on the back surface of the printed skin, the facial deformation becomes natural when motor power was added. Specifically, we prepared the pilot test model that had a mouth hole on the printed skin to simulate the movement of a mouth and two eyes. Two kinds of skins of the same thickness were printed, one was printed with multiple annular ridges with a $1 \mathrm{~mm}$ thickness around them mouth hole, imitating the fibrous shape of the orbicularis oris and the orbicularis oculi muscle, and another one was printed without ridges. When torques were applied to those models, the opening amounts were almost the same, but unnatural deformation occurred above and below the mouth in the model without annular ridges. In the skin without annular ridges, the area around the mouth was deformed evenly. In contrast, in the model with annular ridges, the rigidity increases in the radial direction around the mouth and a deformation shapes along annular ridges. In this way, it was confirmed that the deformation of the skin surface can be adjusted by providing structures with ridges on the back side of the skin.

Using a multi-material 3-D printer, we printed the android head and connected it to the actuator unit which has lined electric motors to actuate the face. We also designed multiple annular grooves on the back of the skin. The motor commands were generated based on the motion capture data of human facial expressions. We observed the same mouth and eyelids movements as in the original human expression.

In Fig. 9, we show the comparison of Degrees of Freedom (DoF) per head volume of the android head and the recently developed androids (Affetto [17], [15], HRP-4C [18], Ibuki [13], and Sophia [19]). The horizontal axis indicates the head volume and the vertical axis indicates DoF in the head (including the neck joints). Whereas, the head volume was defined as the cube of the vertical length of the face as the representative scale. We also plotted data for a 13-year-old Japanese boy [20], assuming the number of muscles is equal to DoF. The straight line in the figure connects the origin with the point of Android Head or the point of the boy and the gradients of these lines represent the density of DoF. Here, it is suggested that the possibility that android printing realizes a large DoF design equal to or higher than that of humans (the red region in Fig. 9).

Our current android head has several limitations. First, we had to design the skin structure manually. It is necessary to design the optimal thickness to have a rigidity in order to give effective facial expressions with limited forces. Second, it is also necessary to design connection point arrangements that can express characteristic facial expressions depending on the individual. Third, the skull-like shell structures on the backside of the skin were not implemented. For example, adding the mandible-skeleton shell is expected to enable greater mouth movements. We think that it is effective to use the numerical calculation such as the finite element method for those design processes. We also expect more efficient driving by optimally designing the number of connection points, motor arrangements and link lengths. In our method using 3-D printing, the design process is mainly done on CG data, so it is suitable with the above numerical calculation method.

Some research directions remain to realize practical application in the future. In order to print the whole body of an android with a multi-material 3-D printing, it is essential to implement both touch sensors on the skin and higher power actuators. To add touch sensors, conductive carbon material 


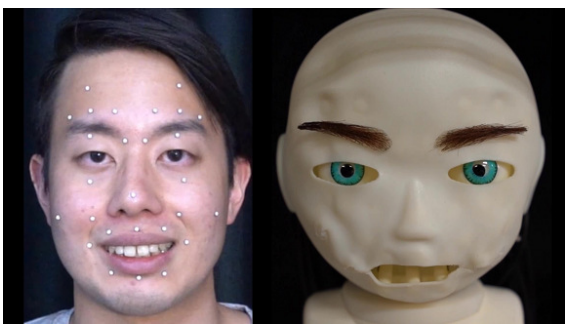

(a)

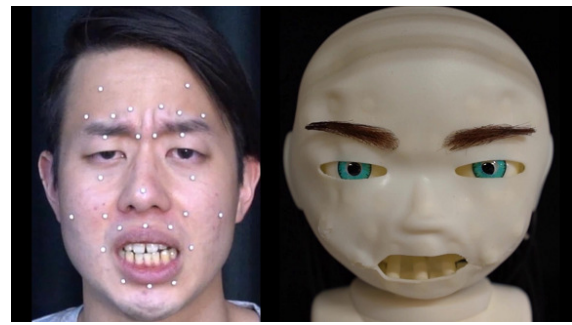

(b)

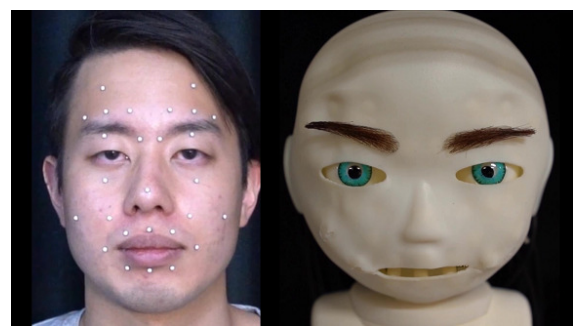

(c)

Fig. 8. The result of three type facial expressions. Left: original human facial expression, right: android head expression.

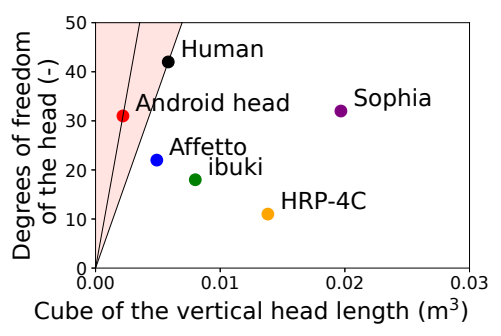

Fig. 9. Comparison of DoF per volume of recent androids

can be used as the printing material, it is possible to detect a contact area on the skin surface. In addition, fluid actuators can be also printed to combine rigid and flexible materials.

\section{CONCLUSION}

We presented the concept of Android Printing, which is a multi-material 3-D printed android head for the initial model of a 3-D printing android. Firstly, we created pilot test models with / without ridges on the skin and we confirmed that ridged skin enables more natural deformation. Secondly, we designed and fabricated a 3-D printed android head with 31 connection points. The skin and link mechanism were printed at once and were connected to an actuator unit combining several electric motors. Lastly, we recreated several facial expressions with the android head based on human facial movements to confirm the feasibility of our concept. In future work, we will add touch sensors to the skin and high power actuators to realize a whole body printed android.

\section{ACKNOWLEDGMENT}

The authors would like to thank Dr. Huthaifa Ahmad for recording the motion capture data.

\section{REFERENCES}

[1] L. D. Riek and R. N. M. Watson, "The age of avatar realism," IEEE Robotics Automation Magazine, vol. 17, no. 4, pp. 37-42, 2010.

[2] A. Kristoffersson, S. Coradeschi, and A. Loutfi, "A review of mobile robotic telepresence," Advances in Human-Computer Interaction, vol. 2013, 2013.

[3] K. Tanaka, N. Yamashita, H. Nakanishi, and H. Ishiguro, "Teleoperated or autonomous?: How to produce a robot operator's pseudo presence in hri," in 2016 11th ACM/IEEE International Conference on HumanRobot Interaction (HRI). IEEE, 2016, pp. 133-140.

[4] K. Tanaka, H. Nakanishi, and H. Ishiguro, "Comparing video, avatar, and robot mediated communication: pros and cons of embodiment," in International conference on collaboration technologies. Springer, 2014, pp. 96-110.
[5] M. Natarajan and M. Gombolay, "Effects of anthropomorphism and accountability on trust in human robot interaction," in Proceedings of the 2020 ACM/IEEE International Conference on Human-Robot Interaction, 2020, pp. 33-42.

[6] S. Nishio, H. Ishiguro, and N. Hagita, "Geminoid: Teleoperated android of an existing person," Humanoid robots: New developments, vol. 14, pp. 343-352, 2007.

[7] G. Langevin. (2012) Inmoov - open source 3d printed life-size robot. [Online]. Available: https://inmoov.fr/

[8] M. Lapeyre, P. Rouanet, J. Grizou, S. N'Guyen, A. Le Falher, F. Depraetre, and P.-Y. Oudeyer, "Poppy: Open source 3d printed robot for experiments in developmental robotics," in 4th International Conference on Development and Learning and on Epigenetic Robotics. IEEE, 2014, pp. 173-174.

[9] J. Kim, A. Alspach, and K. Yamane, "3d printed soft skin for safe human-robot interaction," in 2015 IEEE/RSJ International Conference on Intelligent Robots and Systems (IROS). IEEE, 2015, pp. 24192425.

[10] D. Drotman, S. Jadhav, M. Karimi, P. Dezonia, and M. T. Tolley, " $3 \mathrm{~d}$ printed soft actuators for a legged robot capable of navigating unstructured terrain," in 2017 IEEE International Conference on Robotics and Automation (ICRA). IEEE, 2017, pp. 5532-5538.

[11] Z. Faraj, M. Selamet, C. Morales, P. Torres, M. Hossain, and H. Lipson, "Facially expressive humanoid robotic face," HardwareX, p. e00117, 2020.

[12] R. Ekman, What the face reveals: Basic and applied studies of spontaneous expression using the Facial Action Coding System (FACS). Oxford University Press, USA, 1997.

[13] JST ERATO Ishiguro Symbiotic Human-Robot Interaction Project. (2018, July) Improvement of humanlike conversations in humanoid robots - development of a hild-like android with the ability to move -. [Online]. Available: https://www.jst.go.jp/pr/announce/20180731-2/ index_e.html

[14] C. Becker-Asano and H. Ishiguro, "Evaluating facial displays of emotion for the android robot geminoid f," in 2011 IEEE workshop on affective computational intelligence (WACI). IEEE, 2011, pp. 1-8.

[15] H. Ishihara, B. Wu, and M. Asada, "Identification and evaluation of the face system of a child android robot affetto for surface motion design," Frontiers in Robotics and AI, vol. 5, p. 119, 2018.

[16] M. K. Pan, S. Choi, J. Kennedy, K. McIntosh, D. C. Zamora, G. Niemeyer, J. Kim, A. Wieland, and D. Christensen, "Realistic and interactive robot gaze."

[17] H. Ishihara, Y. Yoshikawa, and M. Asada, "Realistic child robot "affetto" for understanding the caregiver-child attachment relationship that guides the child development," in 2011 IEEE International Conference on Development and Learning (ICDL), vol. 2. IEEE, 2011, pp. 1-5.

[18] S. Kajita, K. Kaneko, F. Kaneiro, K. Harada, M. Morisawa, S. Nakaoka, K. Miura, K. Fujiwara, E. S. Neo, I. Hara et al., "Cybernetic human hrp-4c: A humanoid robot with human-like proportions," in Robotics Research. Springer, 2011, pp. 301-314.

[19] Hanson Robotics. (2020) Sophia. [Online]. Available: https://www. hansonrobotics.com/sophia/

[20] K. ASHIZAWA, "The relationships of head length and breadth in tokyo children," Journal of the Anthropological Society of Nippon, vol. 96, no. 1, pp. 61-70, 1988. 